\title{
On the origin of the $\mathrm{C}$ induced $p 4 g$ reconstruction of $\mathrm{Ni}(001)$
}

\author{
Sergey Stolbov, Sampyo Hong, Abdelkader Kara, and Talat S. Rahman \\ Department of Physics, Kansas State University, \\ 116 Cardwell Hall, Manhattan, Kansas 66506, USA
}

\begin{abstract}
First principles calculations of the geometric and electronic structures have been performed for two coverages (0.25 ML and $0.5 \mathrm{ML})$ of $\mathrm{C}$ on $\mathrm{Ni}(001)$ to understand the mechanism of the $\mathrm{Ni}(001)$ reconstruction induced by carbon adsorption. The calculated structural behavior of the system is in a good agreement with experimental observations. The calculated path and energetics of the $c(2 \times 2)-p 4 g$ reconstruction in $\mathrm{C}_{0.5} / \mathrm{Ni}(001)$ is provided. A dramatic reduction of the local electronic charge on adsorbed carbon is found to occur upon the reconstruction that decreases the electron-electron repulsion on $\mathrm{C}$ site. This effect together with the formation of covalent bonds between $\mathrm{C}$ and the second layer Ni atoms, leads to reconstruction of $\mathrm{Ni}(001)$.
\end{abstract}

\section{INTRODUCTION}

Nickel is used as a catalyst for many reactions. The presence of carbon in the reaction environment may lead to atomic adsorption on the metal surface changing the electronic structure and consequently its reactivity ${ }^{1}$. It is thus important to understand the mechanism by which $\mathrm{C}$ adsorbs on $\mathrm{Ni}$ surfaces. On the other hand, this system is an excellent prototype for addressing fundamental issues such as the effects of the adsorption of light elements on the electronic and geometric structures of metal substrate and the character of the chemical bonding between the adsorbate and the surface. It is thus not surprising that the first observation of $\mathrm{C}$ induced reconstruction of $\mathrm{Ni}(001)^{2}$ has attracted much attention of experimentalists and theorists who aim to provide increasingly accurate description and explanation of the nature of this phenomenon 2,3,4,5,6.7.8.9,10,11,12,13,14.

It is well known that when carbon adsorbs on $\mathrm{Ni}(001)$ with coverage less than one third of a monolayer (ML) it occupies hollow sites and does not change the symmetry of the outmost metal layers ${ }^{3}$. As the coverage exceeds $0.33 \mathrm{ML}$, the surface reconstructs to what has become known as "the clock reconstruction"2.3.4. Fig. 1 illustrates schematically these two 
geometries, in both of which $\mathrm{C}$ atoms occupy the fourfold hollow sites. In the reconstructed structure, the topmost $\mathrm{Ni}$ atoms are displaced by alternate clock-wise and counter-clockwise rotation around $\mathrm{C}$, forming a geometry of the $(2 \times 2) p 4 g$ symmetry. A large volume of additional information has also been obtained from more recent experiments. Scanning tunneling microscopy $(\mathrm{STM})^{\frac{3}{3}}$ and photoelectron diffraction $(\mathrm{PhD})^{\frac{4}{\underline{4}}}$ measurements have been performed for non-reconstructed $\mathrm{Ni}(001)$ with the $\mathrm{C}$ coverage less than 0.3ML $\left(\mathrm{C}_{\theta} / \mathrm{Ni}(001)\right.$, $\theta<0.3 \mathrm{ML})$. Although the quantitative results in the two papers differ, both report a small outward radial displacement of the topmost $\mathrm{Ni}$ atoms (Ni1) around the $\mathrm{C}$ adsorbate. On the other hand, for $\theta>0.33 \mathrm{ML}$, results of low energy electron diffraction (LEED) $)^{\frac{5}{}}$ and $\mathrm{PhD}^{4}$ experiments reveal a large $(0.4-0.55 \AA)$ lateral displacement of the Ni1 atoms and penetration of $\mathrm{C}$ atoms into the Ni1 layer that indicate the surface reconstruction.

The nature of this reconstruction has been a subject of much debate in the past two decades. Using a simple force constant model in lattice dynamical calculations. Rahman et al.. .7 have suggested that the reconstruction is driven by phonon softening. Several scenarios for the reduction of specific force constants were presented, including those involving coupling of $\mathrm{C}$ to the $\mathrm{Ni}$ atoms in the second layer $(\mathrm{Ni} 2)^{2.3 .7 .10}$ which seem plausible since $\mathrm{C}$ penetrates the surface during the reconstruction. On the other hand, Terborg et al. $\underline{\underline{4}}$ argued that the reconstruction is caused by C-induced Ni1-Ni1 repulsion rather than by C-Ni2 bonding, a conclusion that has been upheld in the recent band structure calculations ${ }^{14}$. Charge transfer in the system has also been suggested to be a factor controlling the reconstruction $\underline{8.9}$. However, to our knowledge, the distribution of local charges in the $\mathrm{C} / \mathrm{Ni}(001)$ system have not yet been calculated from first principles. It has also been proposed that surface stress $\frac{10.11}{11}$ might be responsible for the reconstruction. Ab initio calculations by Alfe et al. $\frac{12}{}$, however emphasize chemical bonding effects rather than elastic effects in causing the reconstruction. Direct first principles calculations of $\mathrm{C}$ induced surface stress on $\mathrm{Ni}(001)^{13}$ also do not reveal any specific relationship between surface stress and surface reconstruction. Both Alfe et al. $\underline{\underline{12}}$ and Hong et al $\stackrel{13}{\underline{13}}$ have addressed the issue of geometric and electronic structural changes brought about by the adsorption of $0.5 \mathrm{ML}$ of $\mathrm{C}$ on $\mathrm{Ni}(001)$. Both used the plane wave pseudopotential (PWPP) methods with the local density approximation for the exchange-correlation potential. The authors of Ref. 12 compare the local densities of the electronic states (LDOS) for the non-reconstructed and reconstructed structures. However, no distinct tractable modification of LDOS is found upon the reconstruction. In Ref. 13 the 
valence charge density for the non-reconstructed and reconstructed structures is provided, but authors suggest that a more detailed study of the charge density is required.

The nature of the reconstruction of $\mathrm{Ni}(001)$ thus remains to be unclear, and its understanding requires detailed description of the electronic structure, character of chemical bonding and energetics of the system. Since the reconstruction occurs at a certain C coverage $\theta \approx 0.33 \mathrm{ML}$, it is also important to compare and contrast the electronic structures of $\mathrm{C}_{\theta} / \mathrm{Ni}(001)$ with $\theta<0.33 \mathrm{ML}$ and $\theta>0.33 \mathrm{ML}$.

In this paper, we present the results of our calculations of the geometric and electronic structure of $\mathrm{C} / \mathrm{Ni}(001)$ performed for the $0.25 \mathrm{ML}$ and $0.5 \mathrm{ML} \mathrm{C}$ coverages within the density functional theory with the generalized gradient approximation for the exchange-correlation potential. Applying a structural optimization procedure within the PWPP method, we reproduce the reconstruction for $\mathrm{C}_{0.5} / \mathrm{Ni}(001)$ and provide the energetics and path of transition from $c(2 \times 2)$ to $p 4 g$ geometry. The valence charge densities and local valence charges are calculated for the systems under consideration using more accurate full-potential linearized augmented plain wave method. Analyzing modification of these characteristics upon change in the $\mathrm{C}$ coverage and the reconstruction we provide a consistent description of the mechanism of the reconstruction.

\section{COMPUTATIONAL DETAILS}

All calculations presented in this paper have been performed within the density functional theory with the generalized gradient approximation (GGA) for the exchange-correlation functional $!^{15}$. The $\mathrm{Ni}(001)$ surface was modelled by a tetragonal supercell consisting of a 7 layer Ni slab and $11 \AA$ of vacuum. For both $0.25 \mathrm{ML}$ and $0.5 \mathrm{ML}$ carbon coverages we have used $(2 \times 2)$ two dimensional unit cell which included four $\mathrm{Ni}$ atoms per layer. The supercell has also contained two $\mathrm{C}$ atoms (one atom at each side of the $\mathrm{Ni}$ slab) for the $0.25 \mathrm{ML}$ coverage and four $\mathrm{C}$ atoms (two atoms at each side of the slab) for the $0.5 \mathrm{ML}$ coverage. Thus, the supercells have consisted of 30 and 32 atoms for $0.25 \mathrm{ML}$ and $0.5 \mathrm{ML}$ coverages, respectively.

Surface geometry and energetics have initially been calculated using the PWPP method $\underline{16}$ with ultrasoft pseudopotentials $\underline{\underline{17}}$ used for $\mathrm{C}$ and $\mathrm{Ni}$. We set cutoff energies for the plane-wave expansion of $300 \mathrm{eV}$ for both clean and C-covered surfaces and used a 5x5x1 Monkhorst- 
Pack $k$-point mesh in the Brillouin zone sampling 18 . The structures were optimized until the forces acting on each atom converged better than $0.01 \mathrm{eV} / \AA$.

These optimized structures were then used as input for a more detailed analysis of the electronic structure, which includes self-consistent calculations of the valence charge density and local charges of the systems, using the full-potential linearized augmented plane wave (FLAPW) method ${ }^{19}$ as embodied in the computational code Wien2 $\mathrm{k}^{20}$. Optimized structures obtained from PWPP calculations were used as input data for Wien2k which further refined the geometries after a few ionic iterations. be consistent, we have used the same supercells and k-point mesh in the Brillouin zone for the FLAPW calculations as in the PWPP ones. In the FLAPW method, the local charges are calculated through integration over muffin-tin (MT) spheres of radius $R_{M T}$ centered at each atom. To analyze the effect of the adsorbate on these specific quantities the set of $R_{M T}$ should be chosen to be the same for all structures under consideration. Ideally, $R_{M T}$ should be as large as possible without causing the MT spheres to overlap. For inner atoms of the Ni slab, including the Ni2 atoms, a choice of $R_{M T}=1.19 \AA$ is optimal. However, for $\mathrm{Ni1}, R_{M T}=1.005 \AA$ provided more compatibility which the shorter $\mathrm{C}-\mathrm{Ni}$ bond lengths. For $\mathrm{C}$ atoms $R_{M T}=0.773 \AA$ was needed. To keep plane wave cutoff high enough $\left(R K_{\max }=7\right)$ with the reduced $R_{M T}$ 's for the surface atoms, we used basis sets of about $5500 \mathrm{LAPW}$ 's including 256 local orbitals for the surface with 0.25 ML coverage and for the reconstructed surface with 0.5 ML coverage and 2800 LAPW's including 128 local orbitals for the non-reconstructed surface with 0.5 ML coverage.

\section{RESULTS AND DISCUSSION}

\section{A. Carbon induced structural modification of $\mathrm{Ni}(001)$}

\section{Effects of $0.25 \mathrm{ML} C$ on $\mathrm{Ni}(001)$ geometric structure}

To our knowledge, the case of $0.25 \mathrm{ML} \mathrm{C}$ on $\mathrm{Ni}(001)$ has not been studied so far using first principles calculations based on density functional theory. W3e have carried out such calculations of the geometric structure of the system using a structural optimization procedure that minimizes the forces applied to the atoms. We find our results to be in a good agreement with the experimental observations. Namely, the structural optimization does not lead to the $\mathrm{p} 4 \mathrm{~g}$ reconstruction in this system and results in a stable $\mathrm{c}(2 \times 2)$ geometry. 
Even if we initially shift the Ni1 atoms along the p4g reconstruction path, they revert to the $\mathrm{c}(2 \times 2)$ geometry during the optimization. We find that the presence of $\mathrm{C}$ causes radial outward displacement $\Delta r=0.07 \AA$ of the Ni1 atoms from $\mathrm{C}$. This number is in the range of the experimental results, which spread from $\Delta r=0.02 \pm 0.03 \AA$ (for $0.15 \mathrm{ML}$ coverage) $\frac{4}{\underline{4}}$ to $\Delta r=0.15 \pm 0.15 \AA$ (for about $0.2 \mathrm{ML}$ coverage) ) $^{3}$. The calculated equilibrium C-Ni1 separation $d_{01}=0.24 \AA$ is pretty close to $d_{01}=0.21 \pm 0.07 \AA$ obtained for $\mathrm{C}_{0.15} / \mathrm{Ni}(001)$ from $\mathrm{PhD}$ measurements $\frac{4}{3}$.

\section{Effects of 0.5 MLC on Ni(001) geometric structure}

We starts our calculation of $\mathrm{C}_{0.5} / \mathrm{Ni}(001)$ with the perfect $\mathrm{c}(2 \times 2)$ structure, and calculate the total energy $\left(E_{t o t}\right)$ of the system as a function of $d_{01}$. At each step we fix the $\mathrm{C}$ position and let the rest of atoms relax (move any direction). The result of these calculations is shown on the right side of Fig. 2. No reconstruction occurs during structural optimization and a $E_{\text {tot }}$ minimum is found at $d_{01}=0.53 \AA$. Next, we repeat the procedure, but starting with the surface Ni atoms (Ni1) initially in the p4g reconstructed geometry $\underline{\underline{4}}$. Since the nickel atoms are free to relax as the $\mathrm{C}$ atoms are lowered, the system finds the stable position of Ni1. We find that although Ni1 positions change during optimization depending on $d_{01}$, the system is kept in the p4g structure.

Since $\mathrm{Ni}$ is a ferromagnetic metal, we perform these calculations for both non-magnetic and spin-polarized ferromagnetic cases. The values of $d_{01}$ and the lateral Ni1 displacement $\Delta x y$ obtained for the equilibrium (minimum $E_{t o t}$ ) structures from these two calculations are listed in Tab. \ together with those measured in experiments. As seen from the table, the spin polarization effect on the geometric structure is negligible and both calculated structures are in very good agreement with the experimental observations. To understand why the magnetic effect on the geometric structure is so small, we calculate local Ni spins in the system by integrating spin density over the spheres of $1.2 \AA$ radius centered at different $\mathrm{Ni}$ atoms. We find that while the spin on the $\mathrm{Ni}$ atoms located at the center of the slab equals $0.69 \mu_{B}$, it is reduced to $0.39 \mu_{B}$ on Ni2 neighboring carbon and diminished to $0.02 \mu_{B}$ on Ni1. This result indicates that strong covalent $\mathrm{C}-\mathrm{Ni}$ bonding suppresses spin polarization on the $\mathrm{Ni}$ atoms surrounding adsorbed $\mathrm{C}$ and makes it possible to neglect the magnetic effects on the $\mathrm{C} / \mathrm{Ni}(001)$ surface geometric structure. We thus perform all following calculations for 
non-magnetic systems.

The $E_{t o t}\left(d_{01}\right)$ dependence calculated for non-magnetic p4g structure is plotted on the left side of Fig. 2. One can see that the $E_{\text {tot }}$ minimum for the p4g structure is about $0.8 \mathrm{eV}$ lower than that for the $\mathrm{c}(2 \times 2)$ one. At first sight, both dependences shown in Fig. 2 suggest that the system has two energy minima: the local one for the $\mathrm{c}(2 \times 2)$ structure and the global one for the reconstructed p4g structure and there is an energy barrier between them. However, our further consideration shows that this is a wrong conclusion. C adsorbed in the ideal $\mathrm{c}(2 \times 2)$ structure cannot induce forces acting on $\mathrm{Ni}$ atoms along any direction, which breaks the $\mathrm{c}(2 \times 2)$ symmetry. Regular optimization procedures applying the quasi-Newton or conjugate gradient methods are thus not able to lead such a system to reconstruction. In reality, even if the system appears to be in the $\mathrm{c}(2 \times 2)$ structure, atomic vibrations naturally break the symmetry that can cause the forces leading to the reconstruction.

To place these speculations on firmer ground, we perform another calculation starting with a system slightly deviated from the $(2 \times 2)$ symmetry. Namely we start with the quasiequilibrium $c(2 \times 2)$ structure obtained above, but with Ni1 atoms slightly (by $0.01 \AA)$ shifted along the reconstruction path and let all atoms relax. We find that the structural optimization leads $\mathrm{C}_{0.5} / \mathrm{Ni}(001)$ to the equilibrium $\mathrm{p} 4 \mathrm{~g}$ structure with parameters $\left(d_{01}\right.$ and $\left.\Delta x y\right)$ practically the same as obtained in the simulation described above. The system thus undergoes a $\mathrm{c}(2 \times 2)-\mathrm{p} 4 \mathrm{~g}$ transformation without any energy barrier that is in agreement with the conclusion made in Ref. ${ }^{12}$. The path of the Ni1 and C displacements and the changes in $E_{t o t}$ obtained during the optimization are shown in Fig. 3. One can see that the Ni1 lateral displacement and $\mathrm{C}$ normal shift happen simultaneously, although first Ni1 move faster than $\mathrm{C}$, then, after $\Delta x y$ reaches the value of about $0.4 \AA$, it changes slow, while $d_{01}$ sharply decreases by $0.3 \AA$. At the first stage of the reconstruction the total energy of the system substantially decreases, while at the second stage $E_{t o t}\left(d_{01}\right)$ is flat.

\section{B. Electronic Structure of $\mathrm{C} / \mathrm{Ni}(001)$}

Now, when we find that the structural behavior of $\mathrm{C} / \mathrm{Ni}(001)$ obtained from our calculations completely reproduces the experimental observations, we are going to analyze the relationship between the geometric and electronic structures of the system to reveal factors

controlling the clock reconstruction. To this end, using FLAPW method, we calculate the 
electronic structure for two observed systems: $c(2 \times 2) \mathrm{C}_{0.25} / \mathrm{Ni}(001)$ and $p 4 g \mathrm{C}_{0.5} / \mathrm{Ni}(001)$, as well as, for two hypothetical $c(2 \times 2) \mathrm{C}_{0.5} / \mathrm{Ni}(001)$ structures: one with quasi-stable geometry $\left(d_{01}=0.53 \AA\right)$ and another with a reduced C-Ni1 separation $\left(d_{01}=0.38 \AA\right)$. The valence charge densities calculated for these structures are plotted along a plane perpendicular to the surface in Figs. $4-7$. We find strong covalent C-Ni1 bonds to be formed in all four considered structures. These bonds are seen in the figures as "bridges" of electronic density between the $\mathrm{C}$ and $\mathrm{Ni1}$ atoms. The $c(2 \times 2) \mathrm{C}_{0.25} / \mathrm{Ni}(001)$ and $p 4 g \mathrm{C}_{0.5} / \mathrm{Ni}(001)$ structures also have distinct "bridges" between the $\mathrm{C}$ and $\mathrm{Ni} 2$ atoms, whereas both $c(2 \times 2) \mathrm{C}_{0.5} / \mathrm{Ni}(001)$ structures do not. We find thus that the stable structures observed in experiments differ from unstable ones by the presence of extra C-Ni2 covalent bonds. The extra covalent bond naturally reduces the total energy of the system and increases its stability.

Now the question emerges why the C-Ni2 bonds are not formed in $c(2 \times 2) \mathrm{C}_{0.5} / \mathrm{Ni}(001)$. This bond can occur if the C-Ni2 bond length $\left(l_{C-N i 2}\right)$ is short enough. In Tab. II we provide values of $l_{C-N i 2}$ as well as, the C-Ni1 bond lengths $\left(l_{C-N i 1}\right)$ calculated for the four considered structures. As seen from the table, in the stable $p 4 g \mathrm{C}_{0.5} / \mathrm{Ni}(001)$ and $c(2 \times 2) \mathrm{C}_{0.25} / \mathrm{Ni}(001)$, in which distinct $\mathrm{C}-\mathrm{Ni} 2$ covalent bonds are formed, $l_{C-N i 2}$ are much shorter than in the unstable structures. However, as we reduce $l_{C-N i 2}$ in $c(2 \times 2) \mathrm{C}_{0.5} / \mathrm{Ni}(001)$ from $2.33 \AA$ to $2.18 \AA$, the total energy of the system increases (see Fig. 2). To understand the cause of such a behavior, we focus on local charges on carbon. The local charges $Q_{M T}(C)$, calculated by integrating the valence charge density over the C muffin-tin sphere with $R_{M T}=0.773 \AA$, are listed in Tab. III] We find that if $l_{C-N i 2}$ in $c(2 \times 2) \mathrm{C}_{0.5} / \mathrm{Ni}(001)$ is reduced by $0.25 \AA$, $Q_{M T}(C)$ substantially increases. This is caused by reduction of $l_{C-N i 1}$ that takes place when $l_{C-N i 2}$ decreases: as $l_{C-N i 1}$ becomes shorter, neighboring Ni1 atoms compress the electronic charge density on carbon. This should enhance the electron-electron repulsion at the $\mathrm{C}$ atom that may cause the increase in the total energy mentioned above. The reduction of $l_{C-N i 1}$ happens, because in $c(2 \times 2) \mathrm{C}_{0.5} / \mathrm{Ni}(001)$ there is no room for Ni1 outward displacement. As the clock reconstruction happens, $l_{C-N i 1}$ increases and $Q_{M T}(C)$ dramatically decreases. It should be noted that $Q_{M T}(C)$ in stable $c(2 \times 2) \mathrm{C}_{0.25} / \mathrm{Ni}(001)$ is also significantly smaller than in the unstable $c(2 \times 2)$ structures with the 0.5 ML coverage. However, the C-Ni1 bond length is not the only factor controlling $Q_{M T}(C)$ : if the C-Ni2 covalent bond is formed some portion of the valence electron density is transferred to the C-Ni2 covalent "bridge". This is seen from comparison of $Q_{M T}(C)$ in $c(2 \times 2) \mathrm{C}_{0.25} / \mathrm{Ni}(001), p 4 g \mathrm{C}_{0.5} / \mathrm{Ni}(001)$ and 
$c(2 \times 2) \mathrm{C}_{0.5} / \mathrm{Ni}(001)$ with $d_{01}=0.53 \AA$. All three structures have the same $l_{C-N i 1}=1.83 \AA$ (see Tab. II), but different $Q_{M T}(C)$ (see Tab. III). The C-Ni2 covalent bond is not formed in $c(2 \times 2) \mathrm{C}_{0.5} / \mathrm{Ni}(001)$, and $Q_{M T}(C)$ in this system is the largest. On the other hand, $p 4 g \mathrm{C}_{0.5} / \mathrm{Ni}(001)$ has the strongest $\mathrm{C}-\mathrm{Ni} 2$ covalent bond and the lowest value of $Q_{M T}(C)$. We find thus two factors that make the $c(2 \times 2)-p 4 g$ reconstruction favorable in $\mathrm{C}_{0.5} / \mathrm{Ni}(001)$ : formation of $\mathrm{C}-\mathrm{Ni} 2$ covalent bond that reduces the energy of the system and reduction of $Q_{M T}(C)$ that prevents strong electron-electron repulsion on carbon.

Since interaction of dipoles formed in the surface upon adsorption could also affect surface geometry ${ }^{\underline{9}}$, we calculate the work functions $\Phi$ of the systems which reflect change in the dipole moments. We find, however, that $\Phi$ correlates rather with the $\mathrm{C}-\mathrm{Ni1}$ separation than with in-plane displacements of the Ni1 atoms.

In summary, we have performed first principles calculations of the geometric and electronic structures of $\mathrm{C} / \mathrm{Ni}(001)$ with the $\mathrm{C}$ coverages of 0.25 and $0.5 \mathrm{ML}$. The structural behavior of the system obtained from the calculations is in a good agreement with experimental observations. No energy barrier is found on the reconstruction path. We find that formation of the $\mathrm{C}-\mathrm{Ni} 2$ covalent bonds is crucial for stability of $\mathrm{C} / \mathrm{Ni}(001)$. These bonds are present in the stable $c(2 \times 2) \mathrm{C}_{0.25} / \mathrm{Ni}(001)$ and $p 4 g \mathrm{C}_{0.5} / \mathrm{Ni}(001)$ structures and absent in unstable $c(2 \times 2) \mathrm{C}_{0.5} / \mathrm{Ni}(001)$. Apart from formation $\mathrm{C}-\mathrm{Ni} 2$ covalent bond, the $c(2 \times 2)-p 4 g$ reconstruction in $\mathrm{C}_{0.5} / \mathrm{Ni}(001)$ is accompanied by a dramatic reduction of the local electronic

charge on adsorbed carbon that is supposed to decrease the electron-electron repulsion on C site.

\section{Acknowledgments}

Grant from NCSA, Urbana, IL was beneficial in providing computational resources. We acknowledge financial support from NSF, USA, under grant No. CHE-0205064.

1 1. S. Stolbov, F. Mehmood, T. S. Rahman, M. Alatalo, I. Makkonen, P. Salo, Phys. Rev. B 70, $155410(2004)$.

2 J. N. Onuferko, D. P. Woodruff, and B. W. Holland, Surf. Sci. 87, 357 (1979). 
3 C. Klink, L. Olesen, F. Besenbacher, I. Stensgaard, E. Laegsgaard, and N. D. Lang, Phys. Rev. Lett 71, 4350 (1993).

4 R. Terborg, J. T. Hoeft, M. Polcik, R. Lindsay, O. Schaff, A. M. Bradshaw, R. L. Toomes, N. A. Booth, D. P. Woodruff, E. Rotenberg, and J. Denlinger, Surf. Sci. 446, 301 (2000).

5 Y. Gauther, R. Baudoing-Savois, K. Heinz, and H. Landskron, Surf. Sci. 251-252, 493 (1991).

6 T. S. Rahman, H. Ibach, Phys. Rev. Lett. 54, 1933 (1985).

7 T. S. Rahman, Phys. Rev. B 35, 9494 (1987).

8 C. Q. Sun, P. Hing, Surf. Rev. Lett., 6, 109 (1999).

9 C. Q. Sun, Surf. Rev. Lett., 7, 347 (2000).

10 J. E. Müller, M. Wuttig, H. Ibach, Phys. Rev. Lett. 56, 1583 (1986).

11 D. Sander, U. Linke, and H. Ibach, Surf. Sci. 272, 318 (1992).

12 D. Alfe, S. de Gironcoli, and S. Baroni, Surf. Sci. 437, 18 (1999).

13 S. Hong, A. Kara, T. S. Rahman, R. Heid, and K. P. Bohnen, Phys. Rev. B 69, 195403 (2004).

14 J. E. Kirsch, S. Harris, Surf. Sci. 522, 125 (2003).

15 J. P. Perdew and Y. Wang, Phys. Rev. B 45, 13244 (1992).

16 M. C. Payne, M. P. Teter, D. C. Allan, T. A. Arias, and J. D. Joannopoulos, Rev. Mod. Phys., 64, 1045 (1992).

17 D. Vanderbilt, Phys. Rev. B 41, R7892 (1990).

18 H. J. Monkhorst and J. D. Pack, Phys. Rev. B 13, 5188 (1976).

19 M. Weinert, E. Wimmer, and A. J. Freeman, Phys. Rev. B, 26, 4571 (1982); D. Singh, Planewaves, pseudopotentials and the LAPW method, Kluwer Academic Publishing (1994), ISBN 0-7923-9421-7.

20 P. Blaha, K. Schwarz, G. K. H. Madsen, D. Kvasnicka, and J. Luitz, WIEN2k, An Augmented Plane Wave + Local Orbitals Program for Calculating Crystal Properties (Karlheinz Schwarz, Techn. Universität Wien, Austria) 2001. ISBN 3-9501031-1-2.

\section{FIGURE CAPTIONS}

Fig. 1 (Color online). Illustration of the $c(2 \times 2) \mathrm{C}_{0.25} / \mathrm{Ni}(001)$ (upper panel) and $p 4 g \mathrm{C}_{0.5} / \mathrm{Ni}(001)$ (lower panel) geometric structures. 
TABLE I: Comparison of structural parameters of $\mathrm{C}_{0.5}-\mathrm{p} 4 \mathrm{~g} / \mathrm{Ni}(001)$ calculated in this work with those obtained from experiments 4.5 . $\mathrm{NM}$ and FM denote non-magnetic and ferromagnetic calculations, respectively

\begin{tabular}{ccc}
\hline \hline & $d_{01}(\AA)$ & $\Delta x y(\AA)$ \\
\hline $\mathrm{NM}$ & 0.13 & 0.51 \\
$\mathrm{FM}$ & 0.14 & 0.51 \\
Exper. & $0.1-0.12$ & $0.41-0.55$ \\
\hline \hline
\end{tabular}

TABLE II: C-Ni bond lengths calculated for the considered $\mathrm{C} / \mathrm{Ni}(001)$ structures.

\begin{tabular}{ccc}
\hline \hline & $l_{C-N i 1}(\AA)$ & $l_{C-N i 2}(\AA)$ \\
\hline$p 4 g-\mathrm{C}_{0.5}$ & 1.83 & 1.99 \\
$c(2 \times 2)-\mathrm{C}_{0.25}$ & 1.83 & 2.04 \\
$c(2 \times 2)-\mathrm{C}_{0.5}, d_{01}=0.38$ & 1.79 & 2.18 \\
$c(2 \times 2)-\mathrm{C}_{0.5}, d_{01}=0.53$ & 1.83 & 2.33 \\
\hline \hline
\end{tabular}

Fig. 2. Dependence of the total energy versus the C-Ni1 separation calculated for $\mathrm{C}_{0.5} / \mathrm{Ni}(001)$ in $c(2 \times 2)$ and $p 4 g$ geometries. Zero energy corresponds to $\mathrm{C}$ desorption.

Fig. 3. Reconstruction path and energetics calculated for $\mathrm{C}_{0.5} / \mathrm{Ni}(001)$.

Fig. 4 (Color online). Valence charge density calculated for $c(2 \times 2) \mathrm{C}_{0.25} / \mathrm{Ni}(001)$ and plotted along the plane perpendicular to the surface.

TABLE III: C local charges and work functions calculated for the considered $\mathrm{C} / \mathrm{Ni}(001)$ structures.

\begin{tabular}{ccc}
\hline \hline & $Q_{M T}(\mathrm{C})$ & $\Phi(\mathrm{eV})$ \\
\hline$c\left(2 \times 2-\mathrm{C}_{0.5}, d_{01}=0.53 \AA\right.$ & 4.58 & 5.67 \\
$c(2 \times 2)-\mathrm{C}_{0.5}, d_{01}=0.38 \AA$ & 4.67 & 5.55 \\
$p 4 g-\mathrm{C}_{0.5}, d_{01}=0.13 \AA$ & 4.30 & 5.39 \\
$c(2 \times 2)-\mathrm{C}_{0.25}$ & 4.41 & 5.35 \\
\hline \hline
\end{tabular}


Fig. 5 (Color online). The same as in Fig. 4, but for $p 4 g \mathrm{C}_{0.5} / \mathrm{Ni}(001)$.

Fig. 6 (Color online). The same as in Fig. 4, but for $c(2 \times 2) \mathrm{C}_{0.5} / \mathrm{Ni}(001)$ with $d_{01}=0.53 \AA$.

Fig. 7 (Color online). The same as in Fig. 4 , but for $c(2 \times 2) \mathrm{C}_{0.5} / \mathrm{Ni}(001)$ with $d_{01}=$ $0.38 \AA$. 
This figure "fig1.jpg" is available in "jpg" format from: http://arxiv.org/ps/cond-mat/0507344v1 
This figure "fig2.jpg" is available in "jpg" format from: http://arxiv.org/ps/cond-mat/0507344v1 
This figure "fig3.jpg" is available in "jpg" format from: http://arxiv.org/ps/cond-mat/0507344v1 
This figure "fig4.jpg" is available in "jpg" format from: http://arxiv.org/ps/cond-mat/0507344v1 
This figure "fig5.jpg" is available in "jpg" format from: http://arxiv.org/ps/cond-mat/0507344v1 
This figure "fig6.jpg" is available in "jpg" format from: http://arxiv.org/ps/cond-mat/0507344v1 
This figure "fig7.jpg" is available in "jpg" format from: http://arxiv.org/ps/cond-mat/0507344v1 